\title{
Robotic Mitral Valve Surgey Combined with Left Atrial Reduction and Ablation Procedures
}

\author{
Unal Aydin ${ }^{1}$, MD; Onur Sen ${ }^{1}$, MD; Ersin Kadirogullari ${ }^{1}$, MD; Zeynep Kahraman², MD; Burak Onan ${ }^{1}$, MD
}

DOI: $10.21470 / 1678-9741-2018-0297$

\begin{abstract}
Introduction: This study aimed to evaluate the feasibility and efficacy of robotically assisted, minimally invasive mitral valve surgery combined with left atrial reduction for mitral valve surgery and elimination of atrial fibrillation (AF).

Methods: Eleven patients with severe mitral regurgitation, $A F$, and left atrial enlargement who underwent robotic, minimally invasive surgery between May 2013 and March 2018 were evaluated retrospectively. The da Vinci robotic system was used in all procedures. The patients' demographic data, electrocardiography (ECG) findings, and pre- and postoperative transthoracic echocardiography findings were analyzed. During follow up ECG was performed at postoperative 3,6, and 12 months additionally at the $3^{\text {rd }}$ month trans thoracic echocardiography was performed and functional capacity was also evaluated for all patients.
\end{abstract}

Results: All patients underwent robotic-assisted mitral valve surgery with radiofrequency ablation and left atrial reduction. Mean age was $45.76 \pm 16.61$ years; 7 patients were male and 4 were female. Preoperatively, mean left atrial volume index (LAVI) was $69.55 \pm 4.87 \mathrm{~mL} / \mathrm{m}^{2}$, ejection fraction (EF) was $54.62 \pm 8.27 \%$, and pulmonary artery pressure (PAP) was $45.75 \pm 9.42 \mathrm{mmHg}$. Postoperatively, in hospital evaluation LAVI decreased to $48.01 \pm 4.91 \mathrm{~mL} / \mathrm{m}^{2}(P=0.008)$, EF to $50.63 \pm 10.13 \%(P>0.05)$, and PAP to $39.02 \pm 3.11 \mathrm{mmHg}(P=0.012)$. AF was eliminated in $8(72 \%)$ of the 11 patients at the $1^{\text {st }}$ postoperative month. There were significant improvements in functional capacity and no mortality during follow-up.

Conclusion: Left atrial reduction and radiofrequency ablation concomitant with robotically assisted minimally invasive mitral valve surgery can be performed safely and effectively to eliminate $\mathrm{AF}$ and prevent recurrence.

Keywords: Robotic Cardiac Surgery. Left Atrial Enlargement. Atrial Fibrillation.

\begin{tabular}{ll}
\hline Abbreviations, acronyms \& symbols \\
\hline AF & $=$ Atrial fibrillation \\
CPB & $=$ Cardiopulmonary bypass \\
ECG & $=$ Electrocardiography \\
EF & $=$ Ejection fraction \\
LA & $=$ Left atrial \\
LAE & $=$ Left atrial enlargement \\
LVESD & $=$ Left ventricular end systolic diameter \\
LAVI & $=$ Left atrial volume index \\
NYHA & $=$ New York Heart Association \\
PAP & $=$ Pulmonary artery pressure \\
PTFE & $=$ Polytetrafluoroethylene \\
RF & $=$ Radiofrequency \\
TEE & $=$ Transesophageal echocardiography \\
XC & $=$ Cross clamp
\end{tabular}

'Istanbul Mehmet Akif Ersoy Thoracic and Cardiovascular Surgery Training and Research Hospital, Cardiovascular Surgery Department, İstanbul, Turkey. ${ }^{2}$ Istanbul Mehmet Akif Ersoy Thoracic and Cardiovascular Surgery Training and Research Hospital, Anesthesiology Department, İstanbul, Turkey.

This study was carried out at the Istanbul Mehmet Akif Ersoy Thoracic and Cardiovascular Surgery Training and Research Hospital - Cardiovascular Surgery, Istanbul, Turkey.

\section{INTRODUCTION}

Robotic-assisted surgical platforms are the next step in the evolution of minimally invasive endoscopic procedures, and robotic surgery has become increasingly popular over the last two decades ${ }^{[1-5]}$. The main advantages of these systems are less pain, shorter hospital stay, faster recovery, and improved cosmesis. The da Vinci system (Intuitive Surgical, CA, USA) is the most widely used robotic system. In addition to providing three-dimensional imaging and motion-scaled, tremor-free movements, it improves surgeon dexterity and enables ambidextrous instrument usage. Atrial fibrillation (AF) and left atrial enlargement (LAE) are coexisting pathologies of mitral valve disorders ${ }^{[6]}$. Although successful mitral valve reconstruction reduces left atrial pressure, fibrosis and LAE are irreversible ${ }^{[7]}$. Surgical reconstruction of the left atrium aims to restore synchronous left heart functions and reduce the likelihood of AF recurrence ${ }^{[8]}$

Although robotic mitral valve surgery is well documented,

Correspondence Address:

Onur Sen

iD https://orcid.org/0000-0001-6447-8327

Turgut Ozal Bulvari İstanbul Mehmet Akif Ersoy Kalp Hastanesi, Kalp ve Damar Cerrahi Kliniği, 34303, Küçükçekmece, Istanbul, Turkey

E-mail:dronursen@yahoo.com 
there are no reports in the literature concerning the combination of mitral valve surgery, radiofrequency (RF) ablation, and left atrial reduction. Therefore, the aim of this study was to provide further validation of the efficacy and feasibility of robotic-assisted surgical techniques for this combined procedure.

\section{METHODS}

Between May 2014 and March 2018, a total of 440 patients underwent cardiac operations (due to coronary artery disease, valve disease, atrial septal defect, and cardiac tumors) using the da Vinci robotic system. Mitral valve surgery was performed on 122 (28\%) of those patients, 11 (9\%) of whom were diagnosed with severe mitral regurgitation, AF, and LAE. Data pertaining to these 11 patients were analysed retrospectively (Table 1). Informed consent was obtained from the patients and the study was approved by a local ethics committee. All patients underwent transthoracic and transesophageal echocardiography, coronary angiography, and Doppler ultrasound of the femoral arteries

Table 1. Baseline and datas.

\begin{tabular}{l|c}
\hline Age (mean \pm standard deviation) & $45.7 \pm 16.6$ \\
\hline Sex (Male/female) $(n)$ & $07-4$ \\
\hline Diabetes Mellitus $(n)$ & 3 \\
\hline Hypertension ( $n)$ & 4 \\
\hline COPD (n) & 0 \\
\hline Renal failure $(n)$ & 0 \\
\hline Previous Stroke $(n)$ & 0 \\
\hline Atrial fibrillation & 11 \\
\hline Left atrial thrombus & 1 \\
\hline
\end{tabular}

$\mathrm{COPD}=$ Chronic obstructive pulmonary disease

Table 2. Echocardiography datas.

\begin{tabular}{l|c}
\hline Mitral valvular pathology & \\
\hline \multicolumn{1}{|c|}{ Myxomateous degeneration $(\mathrm{n})$} & 6 \\
\hline Ischemic regurgitation $(\mathrm{n})$ & 0 \\
\hline Functional reg $(\mathrm{n})$ & 0 \\
\hline Rheumatic reg. $(\mathrm{n})$ & 5 \\
\hline Tricuspid reg. $(\mathrm{n})$ & 3 \\
\hline Ejection fraction & $54.62 \pm 8.27$ \\
\hline LVEDD $(\mathrm{cm})$ & $5.76 \pm 0.35$ \\
\hline LVESD $(\mathrm{cm})$ & $4.08 \pm 0.40$ \\
\hline meanPAP $(\mathrm{mmHg})$ & $45.75 \pm 9.42$ \\
\hline LAVI $\left(\mathrm{mL} / \mathrm{m}^{2}\right)$ & $69.55 \pm 4.87$ \\
\hline
\end{tabular}

$\mathrm{LAVI}=$ left atrial volume index; $\mathrm{LVEDD}=$ left ventricle end diastolic diameter; LVESD=left ventricle end sistolic diameter; $\mathrm{PAP}=$ pulmonary artery pressure during preoperative evaluation period. Patients with coronary artery disease, severe peripheral artery disease, sternal or right thoracic reoperations, and emergency surgeries were excluded. However, patients with tricuspid valve disease were included in the study. Extensive pleural adhesions, poor pulmonary function, poor ventricular function, aortic regurgitation, and pectus excavatum are relative contraindications for robotic mitral valve

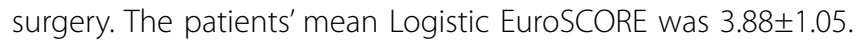
Criteria for undergoing mitral valve surgery were presence of symptomatic chronic severe mitral regurgitation (vena contracta $>0.5 \mathrm{~cm}$, regurgitant volume $>60 \mathrm{ml}$ per beat) with an ejection fraction (EF) $>30 \%$ and left ventricular end systolic diameter (LVESD) $<55 \mathrm{~mm}$, or asymptomatic severe mitral regurgitation with EF $<60 \%$ and LVESD $>40 \mathrm{~mm}$ (Table 2). Surgical RF ablation was performed in patients with persistent AF for more than 7 days despite antiarrhythmic drug therapy. Patients with left atrial diameter $>5.5 \mathrm{~cm}$ also underwent left atrial reduction. The left atrial anteroposterior, mediolateral, and superoinferior diameters were measured by the same cardiologist. These values were used to determine left atrial volume and calculate left atrial volume index (LAVI).

\section{Robotic System Setup}

A left radial artery catheter was placed, and general anesthesia was induced, followed by placement of a single-lumen endotracheal tube and transesophageal echocardiography (TEE) probe. After surgical cannulation of the femoral artery and vein, the jugular and femoral vein catheters were connected to each other, then both arterial and venous cannulas were connected to the cardiopulmonary circuit. The da Vinci robotic system was docked, and the robotic arms were deployed to the designated intrathoracic space (Figure 1). Cardiopulmonary bypass (CPB) was initiated and pericardiotomy was performed, being careful to avoid the phrenic nerve. The pericardium was suspended using retraction sutures. The anterolateral surface of the ascending aorta was sutured with 5-0 PTFE suture for cardioplegia cannula fixation. This cannula can also be used as an aortic root vent. The

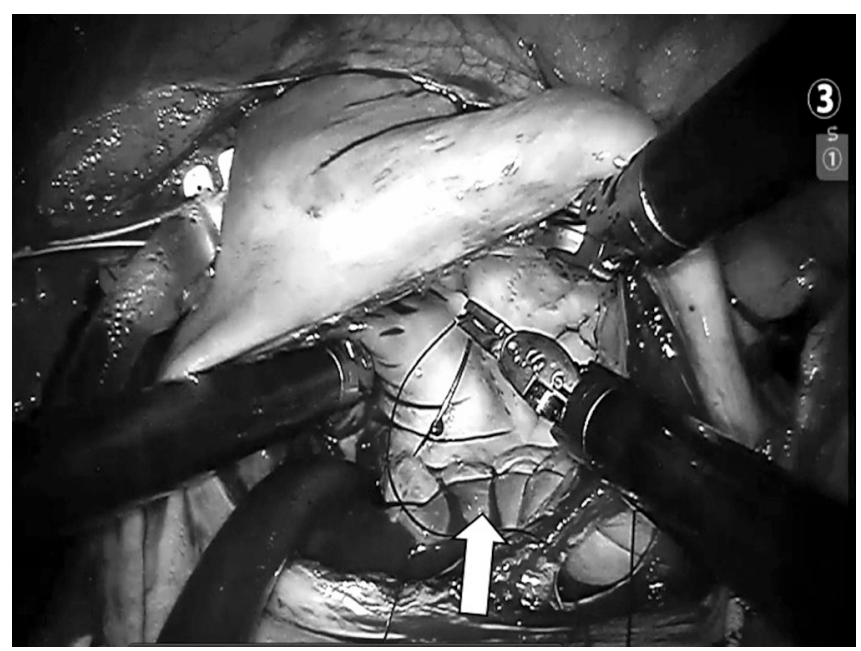

Fig. 1 - Robotic left atrial reduction procedure. 
ascending aorta was occluded using a Chitwood transthoracic aortic cross-clamp (Aesculap Inc. Corporate Parkway Center Valley, PA, USA) and antegrade crystalloid Brethschneider cold cardioplegia was used to arrest the heart.

\section{Surgical Technique}

After arresting the heart, the atrial groove was dissected, and pulmonary veins identified. Left atriotomy was performed and an atrial retractor was used to expose the mitral valve. Mitral valve anatomic configuration, pathology of the valve, appendage, and pulmonary veins were evaluated. The Cox Maze IV procedure was used for creating only left atrial lesion set with RF ablation using a monopolar ablation catheter (Medtronic Cardioblate ${ }^{\circledR}$, Minneapolis, MN, USA). In this manner, RF ablation lines consisted of bilateral pulmonary vein isolation, pulmonary vein roof and floor connecting lesions, lesion from left superior pulmonary vein and atrial appendage, and lesion from inferior atriotomy to mitral valve annulus.

The left atrial appendage was occluded by suturing internally. Left atrial reduction was performed using the posterior atrial plication technique, in which the atrial lines between posterior mitral annulus and circumflex artery was plicated (Figure 1) by continuous suturing. Following mitral annulus suturing, the patient-side surgeon sutured the prosthesis in order and transferred the valve to the left atrium. The robotic arms were positioned the valve at the annulus and a Cor-Knot suture device (LSI Solutions, Victor, NY, USA) was used to securely knot the sutures. Mitral valve repair procedures included neochorda implantation, chordal shortening, or leaflet sliding plasty. Mitral anterior annulus diameter was evaluated, and a rigid ring was implanted in routine fashion (Medtronic Profile 3D ${ }^{\circledR}$, (A, USA). Furthermore, mitral valve replacement was performed by suturing mitral annulus with pledged sutures and valve diameter was evaluated. The prosthetic valve was sutured to anatomic position (St Jude Medical'm Masters Series, Minnesota, USA).

After hemodynamic stabilization, mitral valve functions were evaluated by TEE and CPB was terminated. Robotic arms were removed, and chest drains were placed in the pericardium and right pleura through port incisions. Heparin was reversed and all incisions were closed.

\section{Follow Up}

Every patient was followed up for 3 months exactly because patients were started on class I or III antiarrhythmic drugs and warfarin before hospital discharge. The rhythm was monitored continuously until the discharge, for all patients. If they were in sinus rhythm at the 3rd month, the antiarrhythmic drugs were discontinued. Furthermore, if sinus rhythm was obtained and mitral repair was durable concomitantly by means of echocardiography, anticoagulation was stopped. Follow up conducted by obtaining electrocardiograms studies for all patients at 3, 6 and 12 months and trans thoracic echocardiography studies and functional capacity analysis was performed for all patients at the $3^{\text {rd }}$ month (Tables 3 and 4).

\section{Statistical Analyses}

All analyses were conducted using SPSS 15.0 (SPSS Inc., Chicago, IL, USA). Continuous variables were expressed as mean \pm SD and categorical variables as percentages. Preoperative and postoperative continuous and categorical data were compared with Wilcoxon signed-rank and McNemar tests, respectively. A two-sided $P$ value $<0.05$ was considered statistically significant.

\section{RESULTS}

Mitral valve reconstruction, radiofrequency ablation, and left atrial reduction procedures were performed concomitantly. Left atrial appendage closure was performed in all cases as part of the left atrial reduction procedure. There was no operative or hospital mortality. Bleeding, re-operation, stroke, renal failure or infection did not occur during in hospital and follow up period. Mean CPB time was 147.88 19.12 minutes and mean XC time was $105.75 \pm 20.03$ minutes. Sinus rhythm was achieved in 8 of 11 patients (72.7\%) at postoperative week 1 (Table 5). The mean follow-up time was 16.30 \pm 5.42 months, during which there was no mortality. However, the proportion of patients maintaining sinus rhythm decreased during follow-up. One patient experienced AF recurrence despite antiarrhythmic medication. Postoperative mean LAVI was significantly lower compared to the preoperative value $\left(48.01 \pm 4.91 \mathrm{~mL} / \mathrm{m}^{2}\right.$ vs. $\left.69.55 \pm 4.87 \mathrm{~mL} / \mathrm{m}^{2}, P=0.008\right)$ (Table 3).

Table 3. Statistical analyses of echocardiographic datas.

\begin{tabular}{l|c|c|c}
\hline & Preoperative & Postoperative & $\boldsymbol{P}$ \\
\hline Ejection fraction & $55.22 \pm 7.93$ & $50.63 \pm 10.13$ & 0.059 \\
\hline meanPAP $(\mathrm{mmHg})$ & $47.44 \pm 9.98$ & $39.02 \pm 3.11$ & 0.012 \\
\hline $\mathrm{LAVI}\left(\mathrm{mL} / \mathrm{m}^{2}\right)$ & $69.55 \pm 4.87$ & $48.01 \pm 4.91$ & 0.008 \\
\hline
\end{tabular}

Table 4. Statistical analyses of functional classification.

\begin{tabular}{l|l|l|}
\hline NYHA Class I & 0 & 3 \\
\hline NYHA Class II & 1 & 7 \\
\hline NYHA Class III & 7 & 1 \\
\hline NYHA Class IV & 3 & 0 \\
\hline
\end{tabular}

NYHA=New York Heart Association 
Table 5. Operative and postoperative datas.

\begin{tabular}{|c|c|}
\hline Mitral valve repair (n) & 3 \\
\hline Mitral valve replacement (n) & 8 \\
\hline Maze procedure (n) & 11 \\
\hline Appendage closure (n) & 11 \\
\hline Left atrial reduction (n) & 11 \\
\hline Tricuspid valve repair (n) & 2 \\
\hline CPB time (min) (mean \pm standard deviation) & $147.88 \pm 19.12$ \\
\hline XC time (min) (mean \pm standard deviation) & $105.75 \pm 20.03$ \\
\hline Sinus rythm at hospital discharge (n) & 8 \\
\hline ICU stay (day) (mean \pm standard deviation) & $1.50 \pm 0.53$ \\
\hline $\begin{array}{l}\text { Hospital stay (day) (mean } \pm \text { standard } \\
\text { deviation) }\end{array}$ & $5.22 \pm 1.64$ \\
\hline
\end{tabular}

$\mathrm{AF}=$ atrial fibrillation; $\mathrm{CPB}=$ cardiopulmonary bypass;

$\mathrm{ICU}=$ intensive care unit; $\mathrm{XC}=$ cross clamp

Pre- and postoperative mean New York Heart Association (NYHA) classification indicated significant functional improvement postoperatively ( $3.1 \pm 0.6$ vs. $1.8 \pm 0.6$; $P<0.001$ ) (Table 4).

\section{DISCUSSION}

This study involves the analyses of mitral valve procedures combined with RF ablation and LA reduction procedures via robotic surgery. Mitral valve surgery via robotic-assisted systems enables the surgeon to explore the mitral valve and components in detail without an open procedure. Robotic systems provide three-dimensional visualization and utilize long-shafted endoscopic instruments with seven degrees of freedom. These features enable minimally invasive mitral valve procedures to be performed as successfully as with conventional sternotomy but with reduced trauma, less need for blood and blood products, and shorter intensive care unit and hospital stays ${ }^{[4-6,9]}$. As surgeons use robotic surgical platforms for increasingly complex procedures, it is important to collate clinical data to validate the use of these methods for various surgery types and patient groups. Nifong et al. ${ }^{[10]}$ reported successful surgical management of mitral valve repairs with concomitant biatrial cryoablation via robotic, minimally invasive surgery. In the present study, we report the successful surgical management of mitral valve pathologies with concomitant RF ablation and left atrial reduction through robotic, minimally invasive technique. One concern with increasing the complexity of robotic surgeries may be prolonging $C P B$ and $X C$ times and surgery duration. Robotic surgery is associated with longer $X C$ and CPB times compared to conventional approaches ${ }^{[11]}$. However, it has been reported that the mortality and morbidity rates are similar to the conventional procedures ${ }^{[12]}$. In a 2013 systematic review of robotic mitral surgery by Seco et al. ${ }^{[13]}$, reported CPB times ranged from $106 \pm 22$ to $188.5 \pm 53.8$ minutes and mean $X C$ times ranged from $79 \pm 16$ to $140 \pm 40$ minutes. In the single study that reported CPB and XC times separately for robotic mitral valve surgery with and without cryoablation, CPB times were 188.5 \pm 53.8 and $153.2 \pm 37.7$ minutes and $X C$ times were $130.6 \pm 28.4$ and $116.6 \pm 31.6$ minutes respectively with and without cryoablation ${ }^{[10]}$. The robotic mitral valve surgery we performed consisted of mitral valve repair or replacement, left atrial appendage closure, RF ablation, and left atrial reduction. Nevertheless, our CPB and XC times were $147.88 \pm 19.12$ and $105.75 \pm 20.03$ minutes, respectively. These times are well within the ranges reported in the literature for robotic mitral valve surgery and even below those reported for procedures including ablation. This clearly demonstrates that prolonged CPB and XC times is not an issue with concomitant ablation and left atrial reduction procedures in robotic mitral valve surgery. In experienced centers with refined techniques and skilled robotic teams, operative times should be comparable to those achieved with conventional methods. The determination of the interrelationship of pressure, volume, and wall stress of the cardiac chambers is an important prerequisite for a fundamental understanding of cardiac mechanics. A mathematical model of these mechanics is based on the Law of Laplace $(P=2 \mathrm{HT} / \mathrm{r})^{[14]}$. In this formula, $P$ is pressure, $H$ is wall stress, $T$ is wall thickness, and $r$ is the radius of the chamber. This equation shows that for a given pressure, an increase in radius requires increased wall thickness to maintain a constant wall stress. Furthermore, increased pressure requires greater thickness to maintain a constant wall stress. Consequently, increased left atrial diameter would cause increased wall thickness. The primary mechanism through which wall thickness is increased is fibrous tissue accumulation ${ }^{[15]}$, and left atrial fibrosis plays a major role in the development of atrial fibrillation ${ }^{[16]}$. Based on these data, the surgical strategy used in our practice for patients with mitral valve dysfunction, $A F$, and $L A E$ is to restore normal mitral function via replacement or repair and to perform RF ablation and LA reduction to restore and maintain normal mechanical functions and rhythm. Consequently we preferred only left atrial ablation procedure because no significant difference was mentioned between biatrial and left atrial ablation results in previous studies ${ }^{[17,18]}$. Duration of mitral valve disease is the main factor for LAE and progression of AF, which may be complicated with cerebral and peripheral thromboembolic events. There is no consensus on performing left atrial reduction; however, an association between left atrial size and failure of the maze procedure has been reported previously ${ }^{[19]}$. In another study, AF was eliminated after maze procedure in $100 \%$ of patients with left atrial size $<4.5 \mathrm{~cm}$ and $0 \%$ of patients with left atrial size $>8.5$ $\mathrm{cm}^{[20]}$. In the present study, mean LAVI was $69.55 \pm 4.87 \mathrm{~mL} / \mathrm{m}^{2}$ and sinus rhythm was achieved in 8 patients (72.7\%). We believe that reducing left atrial size is an important factor in the elimination of AF and can facilitate better ventricle function. Our results confirm that significant reductions in left atrial volume can be achieved through a robotic surgical platform.

\section{CONCLUSION}

Our study demonstrates that performing left atrial reduction is feasible through robotically assisted, minimally invasive mitral valve surgery with RF ablation, and yields satisfactory outcomes in the management of mitral valve pathology with concomitant AF and LAE. 
This study is limited by the retrospective, single-center nature of the data and the small number of patients. Another limitation was that the study did not directly compare conventional and robotic mitral valve surgeries combined with left atrial reduction and RF. Nevertheless, our initial results are a novel contribution to the literature in terms of validating robotic-assisted surgical techniques.

\section{No financial support. \\ No conflict of interest.}

\section{Authors' roles \& responsibilities}

UA

Conception and study design; analysis and/or data interpretation; statistical analysis; final approval of the version to be published

OS

Conception and study design; analysis and/or data interpretation; statistical analysis; final approval of the version to be published

EK

Conception and study design; analysis and/or data interpretation; statistical analysis; final approval of the version to be published

ZK Conception and study design; analysis and/or data interpretation; statistical analysis; final approval of the version to be published

BO

Execution of operations and/or trials; manuscript writing or critical review of its content; final approval of the version to be published

\section{REFERENCES}

1. Carpentier A, Loulmet D, Aupecle B, et al. [Computer assisted open heart surgery. First case operated on with success]. C R Acad Sci III [Internet]. 1998 [cited 2019 Apr 27];321(5):437-42. Available from: https://www. ncbi.nlm.nih.gov/pubmed/9766192. French

2. Bush B, Nifong LW, Alwair H, Chitwood WR Jr. Robotic mitral valve surgery-current status and future directions. Ann Cardiothorac Surg. 2013;2(6):814-7. doi:10.3978/j.issn.2225-319X.2013.10.04.

3. Onan B, Aydin U, Kahraman Z, et al. Robot-assisted mitral valve repair with posterior leaflet extension for rheumatic disease. Innovations. 2017;12(1):60-63. doi:10.1097/IMI.0000000000000335.

4. Onan B, Bakir I. Robotic mitral valve replacement in pectus excavatum. J Card Surg. 2016;31(5):306-8. doi:10.1111/jocs.12740.

5. Bakir I, Onan B, Kadirogullari E. Robotically assisted repair of partial atrioventricular canal defect. Artif Organs. 2016;40(9):917-8. doi:10.1111/ aor.12800.
6. Sanfilippo AJ, Abascal VM, Sheehan M, et al. Atrial enlargement as a consequence of atrial fibrillation. A prospective echocardiographic study. Circulation. 1990;82(3):792-7. doi:10.1161/01.CIR.82.3.792.

7. Iwasaki YK, Nishida K, Kato T, Nattel S. Atrial fibrillation pathophysiology: implications for management. Circulation. 2011;124(20):2264-74. doi:10.1161/CIRCULATIONAHA.111.019893.

8. Kamata J, Kawazoe K, Izumoto H, et al. Predictors of sinus rhythm restoration after Cox maze procedure concomitant with other cardiac operations. Ann Thorac Surg. 1997;64(2):394-8. doi:10.1016/S00034975(97)00139-2.

9. Woo YJ, Nacke EA. Robotic minimally invasive mitral valve reconstruction yields less blood product transfusion and shorter length of stay. Surgery. 2006;140(2):263-7. doi:10.1016/j.surg.2006.05.003.

10. Nifong LW, Rodriguez E, Chitwood WR Jr. 540 consecutive robotic mitral valve repairs including concomitant atrial fibrillation cryoablation. Ann Thorac Surg. 2012;94(1):38-42; discussion 43. doi:10.1016/j. athoracsur.2011.11.036.

11. Cao C, Wolfenden H, Liou K, Pathan F, Gupta S, Nienaber TA, et al. A meta-analysis of robotic vs. conventional mitral valve surgery. Ann Cardiothorac Surg. 2015;4(4):305-14. doi:10.3978/j.issn.2225319X.2014.10.05.

12. Bonaros N, SchachnerT, Oehlinger A, et al. Robotically assisted totally endoscopic atrial septal defect repair: insights from operative times, learning curves, and clinical outcome. Ann Thorac Surg. 2006;82(2):68793. doi:10.1016/j.athoracsur.2006.03.024.

13. Seco M, Cao C, Modi P, Bannon PG, Wilson MK, Vallely MP, et al. Systematic review of robotic minimally invasive mitral valve surgery. Ann Cardiothorac Surg. 2013;2(6):704-16. doi:10.3978/j.issn.2225319X.2013.10.18.

14. Moriarty TF. The law of Laplace. Its limitations as a relation for diastolic pressure, volume, or wall stress of the left ventricle. Circ Res. 1980;46(3):321-31. doi:10.1161/01.RES.46.3.321.

15. HanifW, Alex L, SuY, et al. Left atrial remodeling, hypertrophy, and fibrosis in mouse models of heart failure. Cardiovasc Pathol. 2017;30:27-37. doi:10.1016/j.carpath.2017.06.003.

16. Spragg D. Left Atrial Fibrosis: Role in atrial fibrillation pathophysiology and treatment outcomes. J Atr Fibrillation. 2013;5(6):810. doi:10.4022/ jafib.810.

17. Churyla A, Iddriss A, Andrei AC, et al. Biatrial or left atrial lesion set for ablation during mitral surgery: risks and benefits. Ann Thorac Surg. 2017;103(6):1858-65. doi:10.1016/j.athoracsur.2016.10.017.

18. Li H, Lin X, Ma X, et al. Biatrial versus isolated left atrial ablation in atrial fibrillation: a systematic review and meta-analysis. Biomed Res Int. 2018;2018:3651212. doi:10.1155/2018/3651212.

19. Kawaguchi AT, Kosakai Y, Isobe F, et al. Surgical stratification of patients with atrial fibrillation secondary to organic cardiac lesions. Eur J Cardiothorac Surg. 1996;10(11):983-9, discussion 989-990. doi:10.1016/ S1010-7940(96)80401-5.

20. Kosakai Y. Treatment of atrial fibrillation using the Maze procedure: the Japanese experience. Semin Thorac Cardiovasc Surg. 2000;12(1):44-52. doi:10.1016/S1043-0679(00)70016-5. 